\title{
Hydraulic Optimization of Multiphase Pump Based on CFD and Genetic Algorithm
}

\author{
$\mathrm{Hu} \mathrm{Hao}{ }^{1,2}$, Li Xinkai ${ }^{2}$ and $\mathrm{Gu} \mathrm{Bo}^{1}$ \\ ${ }^{1}$ School of Electric Power, North China University of Water Resources and \\ Electric Power, Henan 450011, China \\ ${ }^{2}$ North China Electric Power University, Key Laboratory of CMCPPE Ministry of \\ Education, Beijing 102206, China \\ gb19820915@163.com
}

\begin{abstract}
Impellers of helicon-axial multiphase pump are optimized based on CFD and genetic algorithm. The method mainly includes: CFD numerical calculation, to establish nonlinear relation through neural network, and genetic algorithm optimization extreme. Firstly, the profile of blades is parametric by spline surface and Choose 12 control points as optimization variables. Then, every optimization variable is given optimal dimension. Finally, sample database is got by using standard L27_3_13 orthogonal design table. Next, output values are got by modeling every sample, meshing generation and using CFD numerical calculation. Train neural networks through the database; thus the nonlinear relation between the blade parameter and pump performance parameters is built by applying the nonlinear fitting ability of BP neural networks. Regard the trained neural network as a fitness function of the genetic algorithm and use the characteristic of nonlinear global optimization of genetic algorithm to optimize the multiphase pump. Optimization result shows that the hydraulic efficiency of the multiphase pump is increased by $1.91 \%$.
\end{abstract}

Keywords: multiphase pump; CFD; neural network; genetic algorithm

\section{Introduction}

Oil-gas multiphase pump is key equipment for crude oil shipping and associated gas transportation. With the continuous development of oil field and exploration of submarine oil field, especially the adoption of waterflooding and gas-lift technology, work efficiency and economic profits brought by oil-gas multiphase pump have become a research focus [1-2].

With applying this technology, metering stations can be saved, single pipelines substitute for double ones, and the number of gas-oil separating devices decrease. At the same time, the establishment of oil rigs can be saved during the development of seabed oilfield, the cost of which accounts for 30\% of total capital expenditure [3-4]. Actually, multiphase mixture, such as oil, gas, water and solid impurity co-exist in the process of transportation, when oil-gas multiphase pump is working, which should be sorted out to multiphase pump [5-7]. The multiphase mixture is not treated before transporting, and the content of gas phase and liquid phase in fluid usually exceed the working range of conventional pump and compression pump, so multiphase pumps designed is required to have the function and capability of liquid pump and compression pump [8]. Helicon-axial pumps, which can transport liquid, gas and gas-liquid mixture, are created by drawing lessons from the pump for shipping pure liquid and the compression pump shipping pure gas [9]. Its basic theory [10-11] is: multiphase fluid gets kinetic energy during the fast rotation of impellers. With the help of the diffusing action of guide vane, kinetic energy is converted to pressure energy and the condition of multiphase fluid is adjusted, which 
ensures the next compression unit works properly. Spiral impellers and rectifier force pumped media to run along the axis, preventing gas-liquid media from separating in the runner, which ensures even flowing. Because of the fast rotation of impellers, media entering the impellers are accelerated. These media slow down when passing the rectifier and their kinetic energy is converted to pressure energy. Owing to it, energy is added every time when pumped media pass a unit level. In a vane multiphase pump, the pressure is increased by transporting and converting energy. As core parts of a multiphase pump, impellers (shown in Figure 1) can improve pressure energy of two-phase media and influence its efficiency and stability directly.

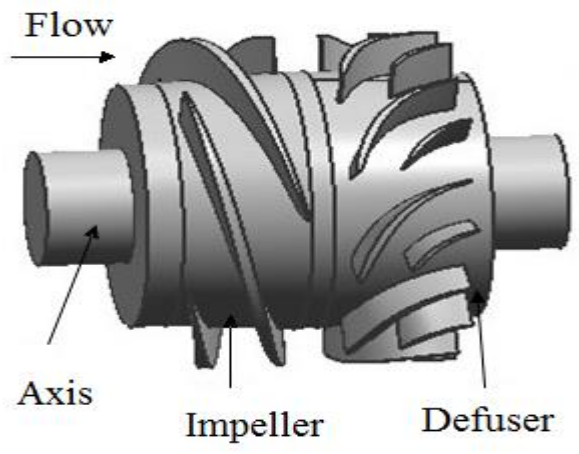

Figure 1. The Model of Impellers of Helion-Axial Multiphase Pump

In January, 1984, Total, IFP and STATOIL cooperated and invested to research "Poseidon" multiphase pump. In 1991, P300 prototype was installed and experimented in Tunisian onshore oil field. In 1994, P301 was installed and applied to Gullfaks rig by Statoil, which symbolized that helicon-axial multiphase pump stepped in the stage of industrialization. Afterwards, helicon-axial multiphase pump is adopted in lots of submarine pressurized mixed transportation projects, such as submarine booster station SMUBS and ELSMUBS created by Framo Engineering, MEPS researched and developed by Mobile and Framo Engineering, Nomad oil production system created by IFP and so on. So far, helicon-axial multiphase pump is widely used in coastal waters [12-14]. At present, there are two ways to design multiphase pumps: one is based on its principle, such as design of multiphase pumps based on quasi-3D controlled velocity moment raised by Li Zengliang, Miao Changshan, et al.; the other is to optimize existing multiphase pumps according to optimization theory, such as optimization design of multiphase pump impeller based on orthogonal design method promoted by Yu Zhiyi et al. Optimization is the development direction of engineering design. This paper tends to adopt optimization theory to raise the pressure and efficiency of multiphase pumps.

This paper tries to forecast the work efficiency of multiphase pumps through CFD, then train BP neural network by sample data gained, based on which and genetic algorithm, the multiphase pumps are optimized.

\section{Optimization Design Process}

Artificial Neural Networks (ANNs) is also called Neural Networks (NNs) for short or named as Connection Model. It is an information processing paradigm, inspired by the central nervous systems of animals [18-21]. BP neural network is a multilayer feedforward neural network. Because the weight is corrected through Backpropagation algorithm, it is also called Back Propagation Network. The structure chart of single-hidden layer neural network is shown in Figure 2. Standard BP training algorithm adopts the steepest descent algorithm to correct weights. During the training process, it begins from a certain point, along the slope of error function, to the minima, with which can minimize the error to 0.To minimize the error to 0 . 


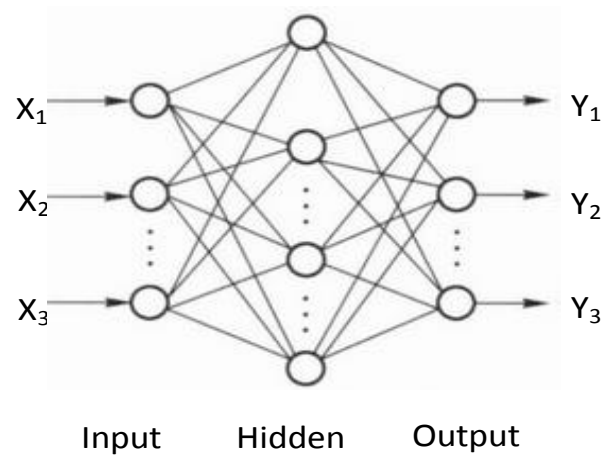

Figure 2. Single Layer BP Neural Network Model

Genetic Algorithm is a computational model imitating hereditary mechanism and evolutionism in nature, finding out the optimization by modeling after natural evolutionary process. Genetic manipulation includes three main genetic operators: selection, crossover and mutation. When the fitness of the best individual hits a given threshold, or the fitness of the best individual and the group doesn't rise, or iterations reach a result settled in advanced.

The process of optimization design main includes, CFD numerical calculation, establishing data relational mapping through neural networks, and genetic algorithm optimization extreme. Design process optimization is shown in Figure 3.

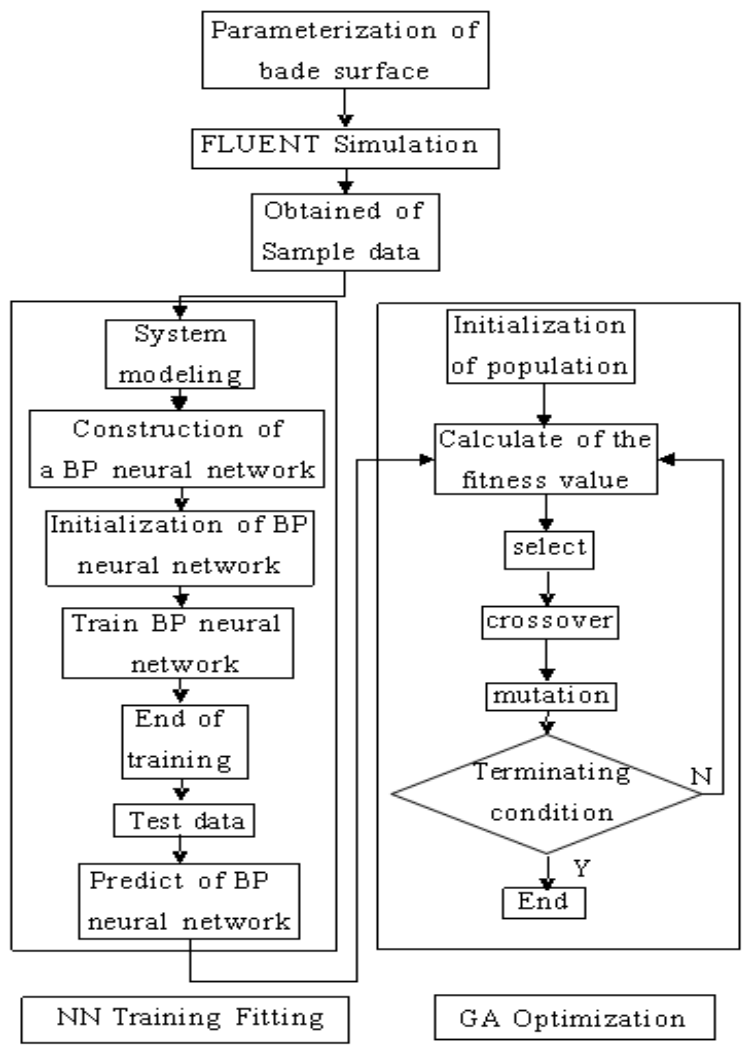

Figure 3. The Optimization Process

\section{Impellers Parameterization}

Pressure surface and suction surface of Impeller blade were parameterized by spline surface. 12 control points were chosen on the surface of an impeller (six points on 
pressure surface and other six on suction surface). According to the movement of control points in the radial direction $\Delta \mathrm{h}$ (positive shift $\Delta \mathrm{h}=2 \mathrm{~mm}$, negative shift $\Delta \mathrm{h}=2 \mathrm{~mm}$, and $\Delta \mathrm{h}=0 \mathrm{~mm}$ )to change molded lines on each flow plane. Control potions were showed in Figure 4. The tables of standard of orthogonal design was used to designs the experiment. In this optimization process with twelve control points and every control point includes three levels $(2,0,-2)$. Because in the standard orthogonal design table no 12 factors and 3 levels case, so L27_3_13 table was chosen to design optimization process, and the 13th factors level is taken as 0 .

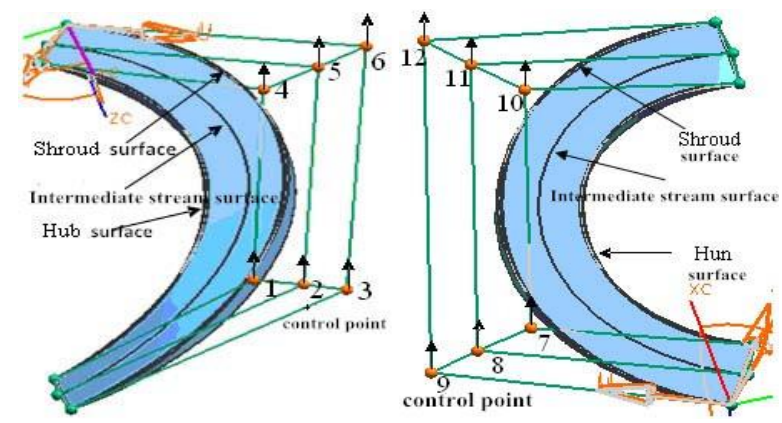

Figure 4. Pressure Surface and Suction Surface Parameterization

\section{CFD Numerical Calculation}

\subsection{Model Establishment}

The research object was showed in Figure 5. In order to save time and computer resources, the paper stimulates the flow field with single channel. Because there are four impellers, take 1/4 of the flow channel surrounding the impeller blades as the physical flow area, and take runner vanes 1/9 of the length of the blades surrounded by an entity as the impeller flow area. The model of multiphase pump and its calculation region are shown in Figure 5.

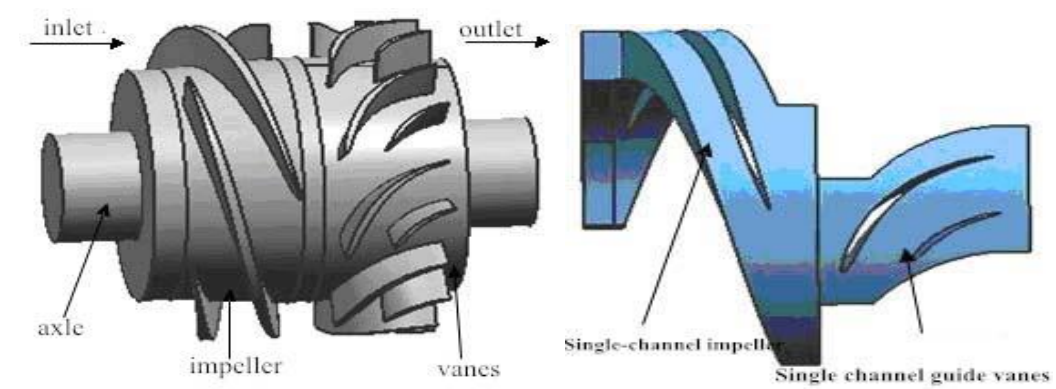

Figure 5. Multiphase Pump Model and Single Channel Model

\subsection{Governing Equation}

Mass conservation equation is a description of fluid into the category of kinematics, namely the mass in the system can never disappear or be destroyed. Momentum conservation equation is a description of equation viscous fluid into the category of dynamics. It is a special form of applying momentum conservation law to fluid, which can be deduced by Newton second law. 
Continuity equation:

$\frac{\partial}{\partial t} \int_{\Omega} \rho d \Omega+\int_{S} \rho \vec{v} \cdot d \vec{S}=0$

Momentum equation:

$\rho \frac{\partial \vec{v}}{\partial t}+\rho(\vec{v} \cdot \vec{\nabla}) \vec{v}=-\vec{\nabla} p+\mu\left[\Delta \vec{v}+\frac{1}{3} \vec{\nabla}(\vec{\nabla} \cdot \vec{v})\right]+\rho \vec{f}$

Energy equation:

$\frac{\partial \rho E}{\partial t}+\vec{\nabla} \cdot(\rho \vec{v} E)=\vec{\nabla} \cdot(k \vec{\nabla} T)+\vec{\nabla} \cdot\left[\left(-p I_{i j}+\tau_{i j}\right) \cdot \vec{v}\right]+\rho \vec{f} \cdot \vec{v}+q_{H}$

\subsection{Governing Equation}

The model of this paper was single channel, so periodic boundary was employed. In order to truthfully reflect the change of flow field and get an accurate result, on the surface of impellers and the angles of inlet and outlet, grids were refined. Grids were coarsened in the steady flow field. Hybrid gird generation is used to control the number of grids so as to obtain a reasonable one. When the number of grids decreases from 500,000 to 360,000, the result changes a little. Therefore, divide the flow field when the number of grids is 360,000 . The direction between the first grid and the wall should meet the needs of $30<y<100$. Mesh generation is shown in Figure 6.
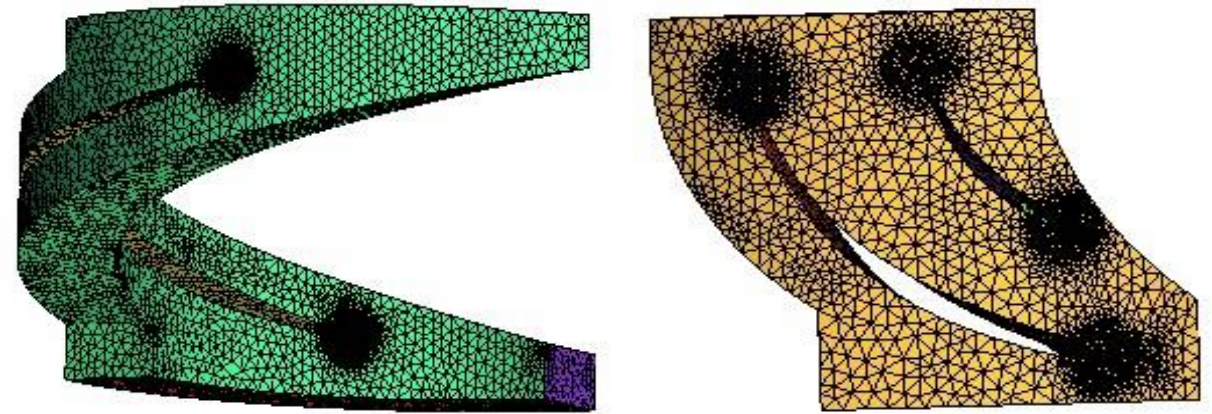

Figure 6. Mesh Generation of Multiphase Pump with Single Channel

\subsection{Two-Phase Flow Model, Equations and Boundary Condition}

Based on Euler multiphase model, finite volume method is adopted to discrete equation, and the second-order upwind scheme was used to discrete the phase. Diffusion term chooses central-difference scheme and Standard $\kappa-\varepsilon$ model to close NS equation; the discrete equations are solved by SIMPLEC.

It is simulated in FLUENT as follows:

Liquid phase is water, and gas phase is air. The gas on the inlet of computational domain is considered well-distributed. The speed of two phases entering the inlet is the same. According to the speed of liquid phase, turbulent energy and dissipation rate are given as follow:

$k_{\text {in }}=0.003 u_{\text {in }}^{2}, \quad \varepsilon_{\text {in }}=C_{\mu} k_{i n}^{3 / 2} / l_{\text {in }}$

$l_{\text {in }}$ refers to mixed length and is assumed as 0.015 long as equivalent diameter of the entrance.

Set the period boundary of impellers and guide vanes as rotating periodic oundary. The outlet of impellers is seen as the outlet of pressure. The inlet of guide vanes is regarded as the inlet of pressure. The interface of impellers and guide vanes 
are mixing planes. According to the operational parameters mentioned above, performance of multiphase pumps before being optimized was calculated and compared with experimental data. With comparison, it shows that the margin of error is no more than $15 \%$ and their curves show the same tendency. Therefore, the numerical calculate method is reliable. Part of the samples of orthogonal design table can be found in Table1.

Table1 Part of the Sample Data of Neural Network

\begin{tabular}{|c|c|c|c|c|c|c|c|c|c|c|c|c|}
\hline \multicolumn{12}{|c|}{ Input sample } & \multirow{2}{*}{$\begin{array}{l}\text { Output sample } \\
\text { Efficiency }(\eta \%) \text { ) }\end{array}$} \\
\hline 1 & 2 & 3 & 4 & 5 & 6 & 7 & 8 & 9 & 10 & 11 & 12 & \\
\hline 2 & 2 & 2 & 2 & 2 & 2 & 2 & 2 & 2 & 2 & 2 & 2 & 0.3119 \\
\hline 2 & 2 & 2 & 2 & 0 & 0 & 0 & 0 & 0 & 0 & 0 & 0 & 0.3223 \\
\hline 2 & 2 & 2 & 2 & -2 & -2 & -2 & -2 & -2 & -2 & -2 & -2 & 0.3437 \\
\hline 2 & 0 & 0 & 0 & 2 & 2 & 2 & 0 & 0 & 0 & -2 & -2 & 0.3543 \\
\hline 2 & 0 & 0 & 0 & 0 & 0 & 0 & -2 & -2 & -2 & 2 & 2 & 0.3394 \\
\hline 2 & 0 & 0 & 0 & -2 & -2 & -2 & 2 & 2 & 2 & 0 & 0 & 0.3439 \\
\hline 2 & -2 & -2 & -2 & 2 & 2 & 2 & -2 & -2 & -2 & 0 & 0 & 0.3516 \\
\hline 2 & -2 & -2 & -2 & 0 & 0 & 0 & 2 & 2 & 2 & -2 & -2 & 0.3140 \\
\hline 2 & -2 & -2 & -2 & -2 & -2 & -2 & 0 & 0 & 0 & 2 & 2 & 0.3280 \\
\hline 0 & 2 & 0 & -2 & 2 & 0 & -2 & 2 & 0 & -2 & 2 & 0 & 0.3468 \\
\hline
\end{tabular}

\section{Nonlinear Fitting of BP Neural Network}

During the optimization, if adopt CFD to calculate all points, there is too much to do and it is unrealistic. Therefore, in this paper optimizes multiphase pumps by employing the characteristic of the nonlinear fitting of BP neural network and nonlinear global optimization of genetic algorithms.

27 groups of sample data were gained through CFD numerical calculation, among which, 20 are training samples and 7 are test samples. Moving distance of the control point was considered as input samples and efficiency was output sample of neural network. Non-linear relationship between the blade parameter of multiphase its external characteristics was established. Prediction error of neural network was tested and the trained neural network was used as a fitness function of the genetic algorithm. The structure chart of one-hidden layer BP neural network is shown in Figure 7.

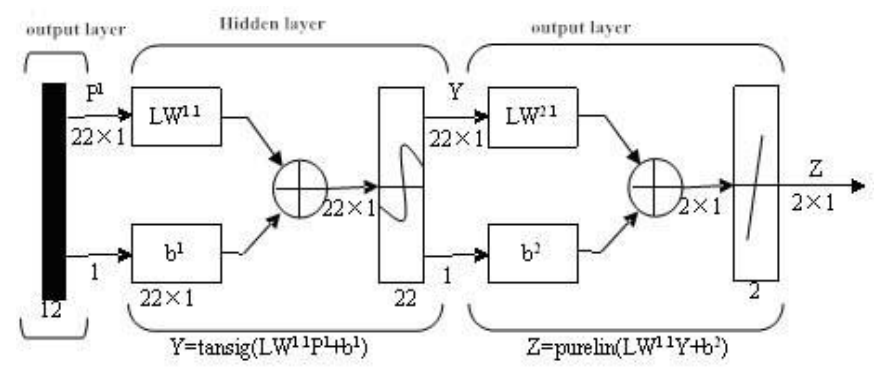

Fig.7 Structure Chart of One-Hidden Layer BP Neural Network

For one neuron will be input from 12 input neurons in the paper, a single hidden layer network was chosen and the number of hidden layer neuron is 22. BP neural network hidden layer transfer function $\operatorname{tansig}()$, output layer transfer function purelin(), network learning algorithm trainlm(). The training steps of network is 1000 and the training accuracy is0.0001. The operation result of BP neural network is shown in Figure8 and 9. 


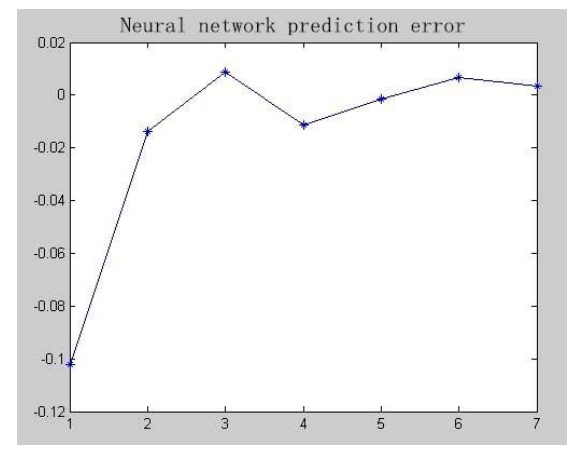

Figure 8. Forecasting Errors of BP Neural Network

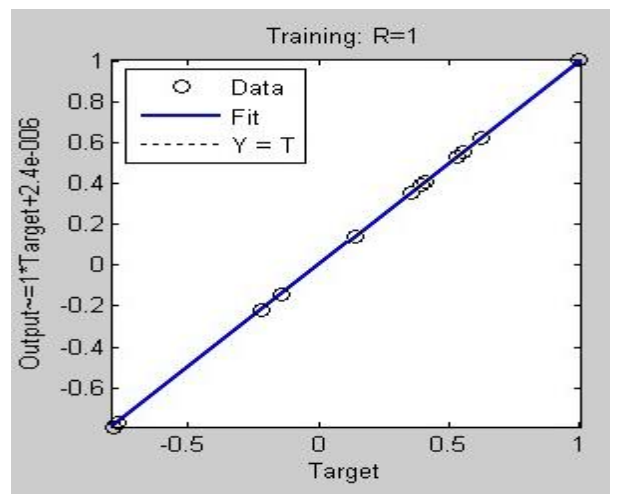

Figure 9. Linear Regression Analysis of BP Neural Network

It can be seen from graph8, in addition to the fact that the error of the first data is larger, and there is little error in the rest. Generalization capability and approximation capability are strong.

\section{Comparative Analysis of Optimal Solutions}

Fitness function of the genetic algorithm was used to get the minimum of a fitness value, so the negative value of output results of neural network was looked at as fitness function. GA parameter settings are maxgen $=100$, sizepop $=50$, pcross $=0.4$, pmutation $=0.2$, bound $=[-2,2]$. The procedure was operated 10 times and the maximum value was used as the optimal solution. The curve of GA fitness value can be seen in graph 4.13

The maximum optimization result of hydraulic efficiency by GA is -0.3657 , and the value of 1-12 control points shown in Figure 11.

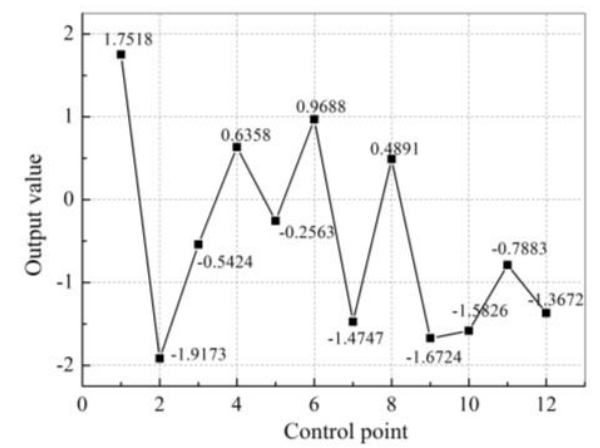

Figure 9. Output Value of Control Point 
In order to evaluate the efficiency of the genetic algorithm, the capacity of the multiphase pump optimized was accessed and forecasted by adopting the same values obtained in the GA to the modeling. The result indicates that the calculation efficiency of the multiphase pump is 0.3671 , in which there is an error of $0.14 \%$ by comparing with genetic algorithm optimal output. Why is there an error between them? The reason is the fitness function of the genetic algorithm is replaced by the neural network, while the neural network has to some degree forecasting errors.

The calculation efficiency of the multiphase pump before optimization is 0.384 , while it is 0.3671 after being optimized. The efficiency of the multiphase pump is increased by $1.91 \%$ maximum and it is obvious that the effect is good.

\section{Conclusions}

(1) A new optimization method was developed about multiphase pump based on CFD and genetic algorithm, and the feasibility of the optimization method was proved.

(2) Firstly the capacity of multiphase pumps were forecasted and accessed based on CFD. Then BP neural network was trained through sample data, and built the nonlinear relation between the blade parameter and the pump performance parameters. Next, the global optimization by genetic algorithms is adopted, and optimized model of multiphase pump blade is established.

(3) The efficiency of the multiphase pump is increased by $1.69 \%$. It proves that the optimization method is feasible and has great significance in engineering research and practice.

\section{Acknowledgments}

This work is supported by Henan provincial key science and technology projects (142102210059). In the same time, there are some people who have done a lot of work for the paper, but they are not listed in the paper, we want to say thanks.

\section{References}

[1] J. Falcimaigne, J. BRAE, Y. Charron, et al., "Multiphase Pumping Achievements and Perspectives", Oil Gas Science and Technology, vol. 57, no.1 (2002) Jan., pp.99-107.

[2] D. M. Link, A. J. schke, G. Schröder, "Multiphase boosting in oil and gas production", The International Petroleum Technology Conference, (2005), pp.10143-10151.

[3] D. F. DalPorto, L. A. Larson, "Multiphase-pump field trials demonstrate practical applications for the technology", Offshore technology conference, Houston, (1996), pp.159-64.

[4] H. Dogru, A. A. Hamoud, S. G. Barlow, "Multiphase pump recovers more oil in a mature carbonate reservoir", Petrol Technology, V. 56, No. 3, (2004) March, pp. 64-72.

[5] P. B. P. Gié, C. Bratu, P. Durando, "Poseidon multiphase pump: field test results", Offshore technology conference, Houston, (1992), pp. 489-502.

[6] J. Salis, C. Marolles, Schachenmann, "Multiphase pumps for marginal oil fields", World Pumps, V. 334, No. 61, (1994), pp. 38-41.

[7] K. Rabiger, T. M. A. Maksoud, J. Ward, et al., "Theoretical and experimental analysis of a multiphase screw pump, handling gas-liquid mixtures with very high gas volume fractions", Exp Therm Fluid, V. 32, No. 9, (2008), pp. 1694-1701.

[8] . P. Li, D. S. Xue, W. Q. Lu, "Some discussions about the design of rotodynamic multiphase pump", Eng Thermolphys, V. 20, (1999), pp. 61-64.

[9] Q. P. Li, "Research on design method of a helico-axial multiphase pump and its experimental studies on performances", 2nd International symposium on multiphase, non-Newtonian and reacting flows, Hangzhou People's Republic of China, (2004), pp. 308-212.

[10] . P. Li, D. S. Xue, H. W. Zhu, "Research on hydraulic design concept of an helicon-axial multiphase pump and its experimental studies on performances", Eng Thermolphys, V. 26, (2005), pp.84-97.

[11] L. Emile, D. Alain, Renault J. Francois, e.g., "Deployment of multiphase pumps on a North Sea field", SPE annual technical conference, New Orleans, (2001).

[12] H. J. Grimstad, 'Subsea multiphase boosting-maturing technology applied for Santos Ltd.'s Mutineer and Exeter field", SPE Asia pacific oil and gas conference, Perth, (2004). 
[13] H. Saadawi, "Operating multiphase helicon-axial pumps in series to develop a satellite oil field in a remote desert location", SPE annual technical conference, Califomia, (2007).

[14] Z. L. Li, C. S. Miao, F. X. Liu, "Design of quasi-3D controlled velocity moment for impeller of multiphase pump", Journal of China University of Petroleum, V. 33, No. 1, (2009), pp. 88-90.

[15] Z. Y. Yu, S. L. Cao, G. Y. Peng, "Hydraulic Design of a Multiphase Rotodynamic Pump Impeller and Test Verification", Chinese Journal of Mechanical Engineering, V. 42, No. 3, (2006), pp. 135-142.

[16] Z. Y. Yu, G. Y. Wang, S. L. Cao, "Extended two-fluid model applied to analysis of bubbly flow in multiphase rotodynamic pump impeller", Front.Mech. Eng. China, V. 4, No. 1, (2009), pp. 53-59.

[17] J. Bode, "Decision support with neural networks in the management of research and development: Concepts and application to cost estimation", Information and Management, V. 34, (1998), pp. 33-40.

[18] J. Bode, "Neural networks for cost estimation: Simulations and pilot application", International Journal of Production Research, V. 38, (2000), pp.1231-1254.

[19] ] Y. J. Xua, Y. B. Tao, C. L. Du, "An integrated micromechanical model and BP neural networkfor predicting elastic modulus of 3-D multi-phase and multi-layer braided composite", Composite Structures, V. 122, (2015), pp. 308-315.

[20] H. S. Wang, Y. N. Wang, Y. C. Wang, "Cost estimation of plastic injection molding parts through integration of PSO and BP neural network", Expert Systems with Applications, V. 40, (2013), pp. 418428.

[21] S. B. Cho, "Towards creative evolutionary systems with interactive genetic algorithm", Applied Intelligence, V. 16, (2002), pp. 129-138.

[22] R. Dawkins, "The evolution of evolvability, artificial life in Interdisciplinary Workshop on the Synthesis and Simulation of Living Systems", Redwood City, (1989).

[23] . J. Grefenstette, "Optimization of Control Parameters for Genetical algorithms", IEEE Transactions on Systems Man and Cybernetics, V. 16, No. 1, (1986), pp. 122-128.

\section{Author}

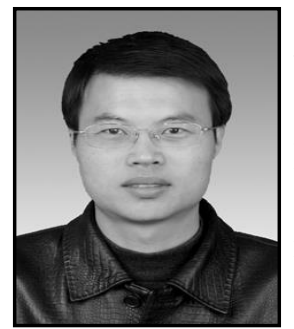

Hu Hao, received the Master degree in North China University of Water Resources and Electric Power in 2008. Currently, he is a lecturer at North China University of Water Resources and Electric power, at same time, he is a PhD student of North China Electric Power University. His interests are in new energy generation technologies. 
International Journal of Grid Distribution Computing Vol. 8, No.6, (2015) 